\title{
A POROUS MEDIUM MODEL FOR PREDICTING THE DUCT WALL TEMPERATURE OF SODIUM FAST REACTOR FUEL ASSEMBLY
}

\author{
Yiqi $\mathrm{Yu}^{1}$, Elia Merzari ${ }^{2}$, Aleksandr Obabko ${ }^{2}$, Justin Thomas ${ }^{1}$ \\ 1. Nuclear Engineering Division, Argonne National labortary, Lemont,IL 60439, United State \\ 2. Mathematics and Computer Science Division, Argonne National labortary, Lemont,IL 60439, United State
}

\begin{abstract}
Porous medium models have been established for predicting duct wall temperature of sodium fast reactor rod bundle assembly, which is much less computationally expensive than conventional CFD simulations that explicitly represent the wire-wrap and fuel pin geometry. Three porous medium models are proposed in this paper. Porous medium model 1 takes the whole assembly as one porous medium of uniform characteristics in the conventional approach. Porous medium model 2 distinguishes the pins along the assembly's edge from those in the interior with two distinct regions, each with a distinct porosity, resistance, and volumetric heat source. This accounts for the different fuel-to-coolant volume ratio in the two regions, which is important for predicting the temperature of the assembly's exterior duct wall. In Porous medium model 3, a precise resistance distribution was employed to define the characteristic of the porous medium. The results show that both porous medium model 2 and 3 can capture the average duct wall temperature well. Furthermore, the local duct wall variations due to different sub-channel patterns in bare rod bundles are well captured by porous medium model 3, although the wire effect on the duct wall temperature in wire wrap rod bundle has not been fully reproduced yet.
\end{abstract}

Keywords: porous medium; duct wall temperature; porosity; resistance.

\section{INTRODUCTION}

Core designers for liquid metal fast reactors have evolved a number of methods for investigating temperature and pressure distribution within these assemblies in order to determine cladding hot spots, assembly bowing and deformation. Experiments are no doubt the most accurate way to create the reliable reference data. Many researchers have carried out experiments for fuel assemblies with wire-wraps. In the early seventies, a benchmark test for a 19pin wire-spacer fuel assembly was performed in Oak Ridge. The measurements for temperature distribution in the duct wall and at the exit of a 19-pin wire spacer fuel assembly were obtained (Fontana (1973), Wantland et al. (1976)). The thermal hydraulic experiments in a 61-rod electrically heated model of an LMFR assembly were performed by Engel et al. (1980) to study the heat transport. Choi et al. (2003) investigated a 217-pin wire spacer fuel assembly to evaluate the performance of existing friction factor correlations. Recently, Sato et al. (2009) reported hydraulic experiments of a 7-pin rod bundle in support of the assessment of the Japanese Sodium Fast Reactor (JSFR) at JAEA. Different experiments are reported by Prakash et al. (2011) to qualify a fuel assembly for the Indian Prototype Fast Breeder Reactor (PFBR). Although an extensive set of wire wrapped rod bundle experiments has been performed all over the world, there are several limitations for the available experimental datasets:

1. Most experimental data is limited to rod bundles with a small number of pins. This data cannot be directly translated to rod bundles with more pins (Collingham et al. (1970)), which restrict their industrial application.

2. Due to geometric or instrumentation limitations, detailed information about both local flow and local temperature fields for one assembly is not available in open literature, not to mention for whole core.

Recent experiments have been proposed to potentially overcome these issues, but the data is not currently available.

\footnotetext{
* Corresponding author. Tel.: +1-630-252-1604.
}

E-mail address: yyu@anl.gov. 
For design purpose, in past years much effort has been made to develop the bundle thermal hydraulic sub-channel analysis codes to yield detailed coolant temperatures for all the sub-channels in the bundle. A sub-channel analysis code MATRA-LMR was developed specially for LMR analysis, the accuracy of which has been confirmed by a benchmark study of the ORNL 19 pin test (Kim et al. (2002)). The subchannel model in the SAS4A/SASSYS-1 code was used to demonstrate a whole plant transient simulation consisting of an unprotected loss-of-flow (ULOF) accident in a 217-pin fuel bundle design (Fanning (2009)).Some other sub-channel codes, such as COBRA-IV-I (Wheeler et.al., 1976; Stewart et al., 1977) and SUPERENERGY-2 (Basehore and Todreas, 1980; Yang and Yacourt, 1995), were developed to perform the similar investigation. However, current whole-core sub-channel models rely on empirically-derived cross-channel mixing terms that do not properly represent the axial dependence of subchannel cross flow. Therefore many numerical researches were performed as a surrogate for experiment to reveal the thermal hydraulic characteristic in wire-wrap rod bundle.

In many existing numerical calculations, CFD simulations have achieved a certain level of maturity to reproduce the temperature and velocity field in bare bundles (Merzari and Ninokata, 2011, Cheng and Yu, 2009, Yu et al., 2011,) and wire wrap bundles assemblies ranging from 7 pins to 217 pins. ANL started performing LES of a single pin (Fischer et al., 2007). Merzari et al. (2010) compare their LES and RANS simulations to the detailed reference DNS data. Pointer et.al.,(2009) performed LES simulations of a 7-pin, a 19-pin, a 37-pin, and finally a 217-pin rod bundle. These simulations show that the bulk swirling reduces importance with increasing bundle size. However, this comes at the cost of an increased flow complexity. Furthermore, a fundamental change in bulk flow behavior between the 19-pin and the 37-pinbundle was observed. Within the 19-pin bundle, the bulk flow in the central sub-channels still shows the influence of the wrapper walls, whereas in the 37-pin bundle, the bulk flow behavior in the central subchannels seems to be decoupled from the influence of the wrapper walls. But the computational cost of these simulations is tremendous. In order to reduce the amount of computational effort but to maintain a reasonable accuracy, a low resolution RANS approach is reported by Hu and Fanning (2010). In this approach they try to simplify the geometrical representation of the wire by either a spiral fin or a momentum source. Although the computational cost is reduced, there is still an incentive to further reduce the cost in order to support design and safety performance analysis efforts. The purpose of the low resolution approaches is to provide more (local) details than typically can be deducted from system thermal hydraulics simulations or sub-channel simulations, while maintaining a reasonable accuracy compared to detailed CFD analyses for a complete fuel assembly which are too costly for engineering purposes.

A low resolution approach can be very useful to study the radial core deformation due to large thermal gradients present in a Sodium Fast Reactor (SFR). These deformations are known to cause important neutronic feedbacks in several SFR designs (Chang et al. 2006). Full core calculations are needed to evaluate this significant safety phenomenon. To simulate correctly the radial core expansion, it is necessary to perform coupled neutronic, structural mechanics and thermal-hydraulic calculations (Mahadevan et al. (2014). In system codes (Wigeland, 1986), this is usually achieved by approximating the structural response using a simplified mechanistic model. The primary driver for radial core expansion is the thermal expansion of the assembly ducts and their contact and interaction with other assemblies, load pads and restraint rings. The structures internal to the assembly are usually ignored and are assumed to slide with regards to the duct.

Recently, the SHARP (Mahadevan et al. 2014) multi-physics modeling capability has been demonstrated for the problem of radial core expansion and bowing in a sodium-cooled fast reactor in the Advanced Burner Test Reactor (ABTR, Chang et al. 2006). The ABTR core presents a complex design including a series of restraint rings, as it is designed to bow outward in response to thermal expansion of the structures in any transient where the core is heating. The fuel pins move apart enhancing neutron leakage and providing a significant negative feedback to the core's reactivity, thus contributing to the passive safety response of the ABTR.

SHARP was used to perform a single integrated simulation of the core undergoing radial thermal expansion while retaining the full geometric detail necessary to model physical phenomena at the continuum scale. This simulation employs the neutronics (PROTEUS), thermal-hydraulics (Nek5000), and structural mechanics (Diablo) simulations simultaneously, with no need for offline perturbation analyses (Mahadevan et al. 2014).

The iteration between the coupled codes is described briefly in Figure 1a. A two level iteration scheme has been employed: including a global iteration and an inner iteration. In the inner iteration the global coupled thermalhydraulic and neurotic problem is solved: Nek5000 and PROTEUS communicate temperature distribution, properties and power density until convergence is reached. In the global iteration the temperature distribution predicted by Nek5000 is transferred to Diablo, which computes deformations due to thermal expansion. After the 
structural mechanics calculations, meshes and properties are thus updated in both Nek5000 and PROTEUS and a new coupled thermal-hydraulic/neutronic solve is performed.

The reactivity feedback from core deformation is therefore predicted explicitly. An example mesh for the restraint ring of ABTR is shown in Figure 1b. Figure 2a presents a sketch of the fuel assembly design for ABTR (Chang et al. 2006 ) with the load pads highlighted. Figure $2 b$ shows the displacements in the y direction magnified 100 times. The thermal-hydraulic model used for this simulation is based on the porous medium methodology described in the following.

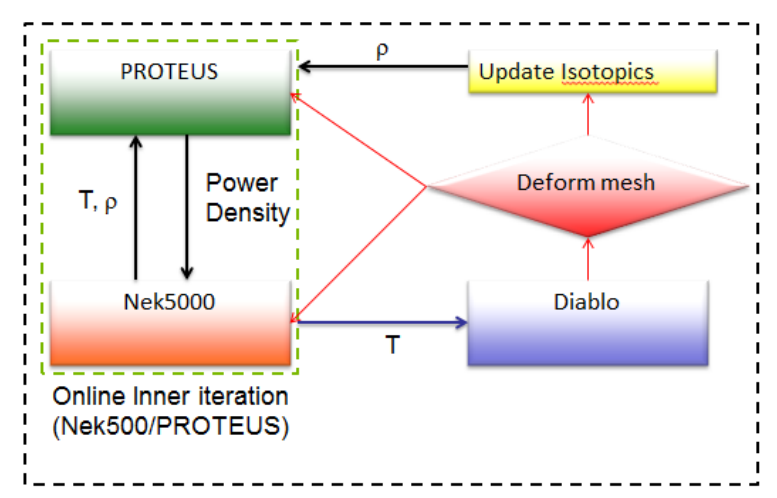

Global iteration

a)

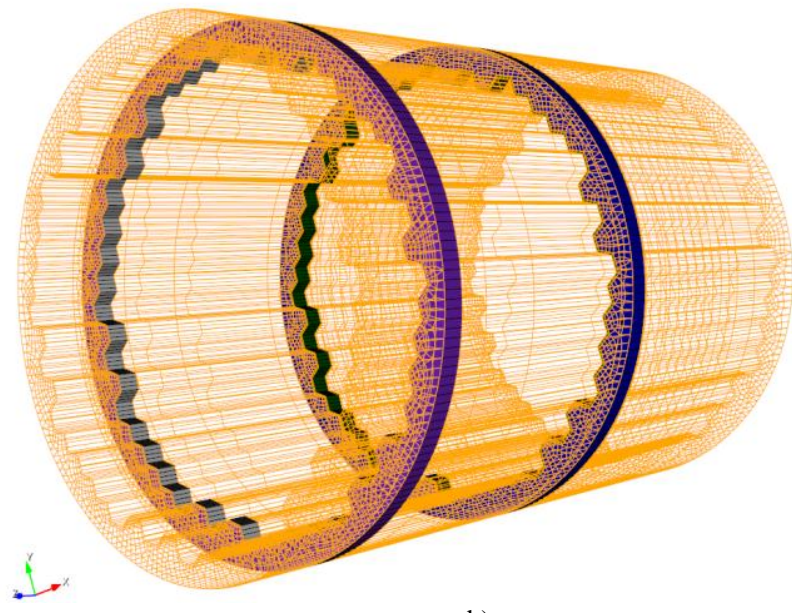

b)

Figure 1 a) Sketch of iteration scheme used for coupled calculation. b) Mesh of restraint ring with gap for the ABTR

The porous medium model is not meant to replace sub-channel analysis or other thermal-hydraulic system codes. It provides an efficient and potentially accurate tool for coupled calculation of radial core expansion. Reference simulations were performed with a CFD model with an explicit representation of the geometry of a 217-pin wirewrapped fuel bundle with commercial CFD software STAR-CCM+. The reference simulation was compared to various models that employ porous media. This work proposes three porous medium models based on the theoretical analysis. The main purpose of this work is to apply porous media model whose solution requires less computational time to calculate the duct wall temperature distribution in wire wrap fuel rod assembly.

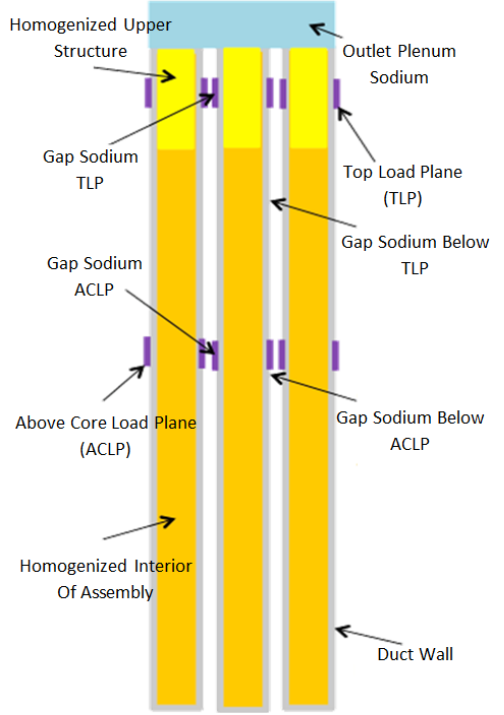

a)

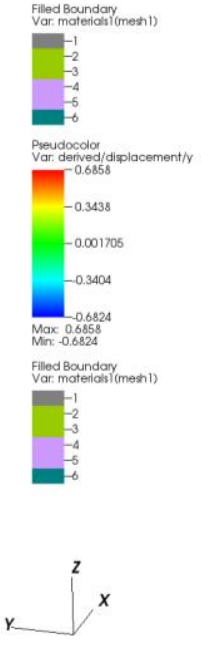

Figure 2 a) Assembly design for the ABTR with load pad b) Magnified (100x) displacements colored by the displacement in the y direction

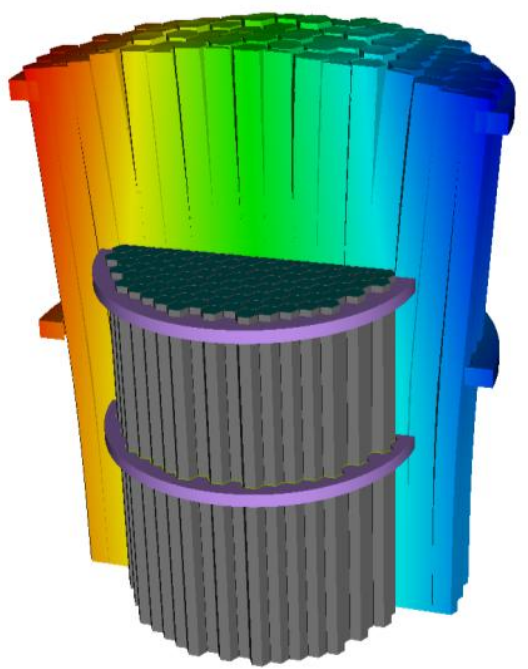

b) 


\section{MODEL DESCRIPTION}

\subsection{CFD MODEL}

The 217 pins ABTR fuel assembly is used as a reference fuel assembly design in this study, the parameters of which are listed in Table 1.The assembly has a relative small coolant flow area with P/D of 1.13. The geometry representation of the contact point between the wire and the pin is simplified to significantly reduce the cell count without further reduction in the mesh resolution, shown in Figure 3. As a result of the high inlet velocity $(4.98 \mathrm{~m} / \mathrm{s})$, the reference case has a high Reynolds number $(50,000)$ based on the rod diameter $(8 \mathrm{~mm})$. In addition, the inlet flow temperature and the rod heat flux for the reference case are $628 \mathrm{~K}$ and $1.14 \mathrm{E} 6 \mathrm{~W} / \mathrm{m} 2$ respectively. An intensive verification study of the turbulence model, mesh radial-to axial aspect ratio, cell count has been carried out to assure the accuracy of the simulation (Yu et al. 2006). Based on the verification study, Realizable k- $\varepsilon$ turbulence model, the polyhedral mesh containing 3.9 million cells with a radial-to-axial aspect ratio of 1:8 are used in the simulation. The solution is well converged as the normalized residual are below $10^{-4}$ with two-layer all-y+ wall formulation, segregated flow solver and the SIMPLE predictor-corrector algorithm.

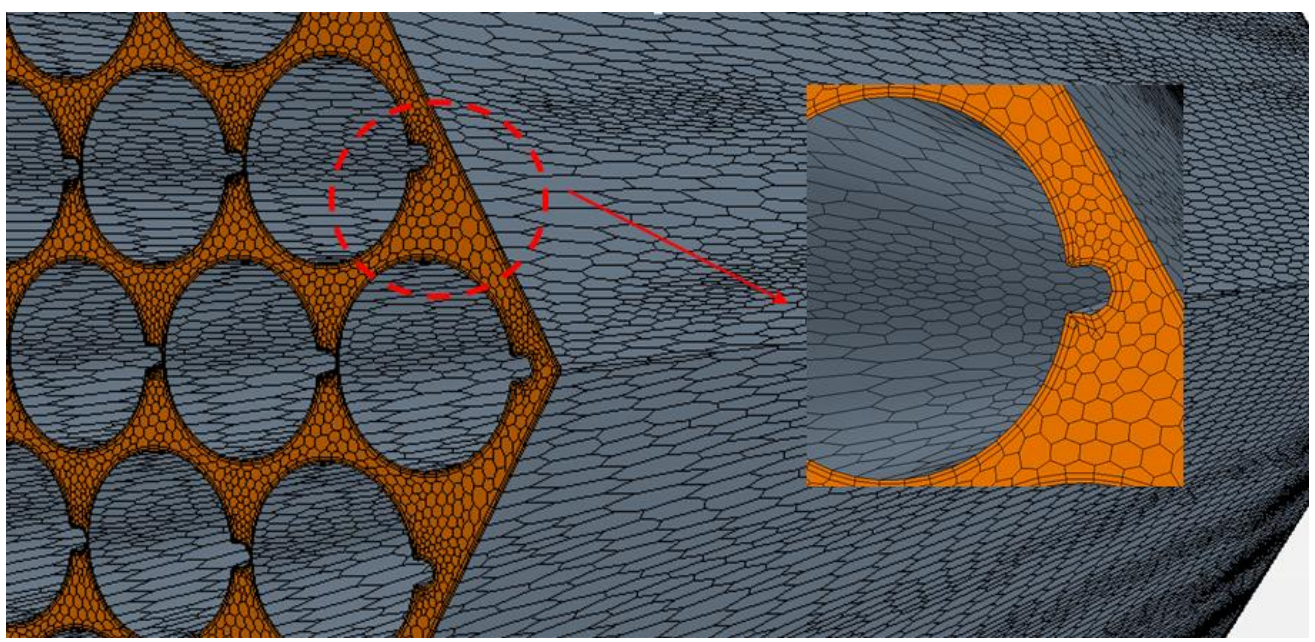

Figure 3 Polyhedral mesh of a 217 pin rod bundle with simplified wire-wrapped pin geometry

Table 1217 pins ABTR fuel assembly geometry information

\begin{tabular}{|c|c|c|}
\hline Parameter & Value & unit \\
\hline number of rods $\left(N_{r}\right)$ & 217 & - \\
\hline rod diameter $\left(D_{r}\right)$ & 8 & $\mathrm{~mm}$ \\
\hline rod pitch $(P)$ & 9.04 & $\mathrm{~mm}$ \\
\hline$P / D$ & 1.13 & - \\
\hline wire wrap diameter & 1.03 & $\mathrm{~mm}$ \\
\hline wire wrap pitch $(H)$ & 203.2 & $\mathrm{~mm}$ \\
\hline
\end{tabular}

\subsection{POROUS MEDIUM MODEL}

The porous medium model imagines the existence of a large number of tightly spaced rods, and treats the rod assembly as a porous medium, much like a bundle of drinking straws that are glued together, approximating it as a continuum. However, the heat transferred from one point to another, at a given plane would be overestimated by this assumption because in actuality the presence of the rods considerably distorts the heat flow path which is termed as "tortuosity" in the open literature (Khan et al. (1975)). As shown in figure 4, the heat flow lines must curve around the rods, which results in an increase in the length of their path. In order to take into account the tortuosity in the porous medium model, the conductivity term is multiplied by the coefficient $\kappa(\kappa<1)$. In principle the value of $\kappa$ can be determined by solving the heat conduction equation $\nabla^{2} \mathrm{~T}=0$ for the configuration shown in Figure 4 . It was 
found that $\kappa$ is nearly equal to the minimum lateral porosity $\lambda_{L}$ of the rod bundle, i.e. $\lambda_{L}=1-\left(D_{r} / P\right)$, where $D_{r}$ is rod diameter and P is rod pitch (Khan et al. (1975)).

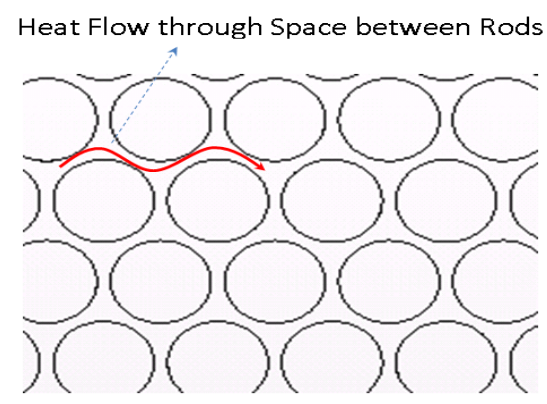

Figure 4 Heat flow path in rod bundle geometry

Different authors provide us with friction factor correlations for wire wrapped rod bundles based on a particular set of experimental data. These correlations are very good for the prediction of pressure drop for wire wrapped rod bundle within the parameter range for which they were derived based on certain fluid and certain rod bundle parameters. According to the parameters in this study, the present porous medium model use Rehme's (1973) correlations to calculate the pressure drop in the rod bundle assembly.

$$
\Delta p=\mathrm{f}\left(\frac{H}{D e}\right) 0.5 \rho v^{2}
$$

where $f$ is the friction factor, De is the hydraulic diameter and $\mathrm{H}$ is the duct length. $f$ is calculated using the following equations:

$$
\begin{gathered}
\mathrm{f}=\left(\frac{64}{R e} F^{0.5}+\frac{0.0816}{R e^{0.133}} F^{0.9335}\right) \frac{N_{r} \pi\left(\mathrm{D}_{r}+\mathrm{D}_{w}\right)}{S_{t}} \\
\mathrm{~F}=\left(\frac{\mathrm{P}_{\mathrm{t}}}{\mathrm{D}_{\mathrm{r}}}\right)^{0.5}+\left[7.6 \frac{\left(\mathrm{D}_{\mathrm{r}}+\mathrm{D}_{\mathrm{w}}\right)}{\mathrm{H}}\left(\frac{\mathrm{P}_{\mathrm{t}}}{\mathrm{D}_{\mathrm{r}}}\right)^{2}\right]^{2.16} \\
\mathrm{P}_{\mathrm{t}}=\mathrm{D}_{\mathrm{r}}+1.0444 \mathrm{D}_{\mathrm{w}}
\end{gathered}
$$

Where $\mathrm{Nr}$ is rod number, $\mathrm{Dw}$ is wire diameter, $\mathrm{S}_{\mathrm{t}}$ is total wetted perimeter. In STAR-CCM+, the pressure drop is specified as follows:

$$
-\frac{\mathrm{dp}}{\mathrm{H}}=\mathrm{P}_{\mathrm{v}} \mathrm{v}+\mathrm{P}_{\mathrm{i}} \mathrm{v}^{2}
$$

where $P_{v}$ is the viscous resistence and $P_{i}$ is the inertial resistance. Since the porous resistance is a quadratic function of flow velocity, only the inertial resistance tensor is specified. In order to restrict the flow in the directions perpendicular to the primary direction, a resistance coefficient that is 2-3 orders of magnitude larger than primary coefficient is specified. Choosing a larger resistance does not affect the flow but can adversely affect the convergence characteristics. Therefore the viscous resistance tensor is chosen to be:

$$
\left[\begin{array}{ccc}
\mathrm{P}_{\mathrm{i}} & 0 & 0 \\
0 & 100 \mathrm{P}_{\mathrm{i}} & 0 \\
0 & 0 & 100 \mathrm{P}_{\mathrm{i}}
\end{array}\right]
$$

Calculated with the correlations mentioned above, the pressure drop obtained in the porous medium is 2.96E+04 Pa, which agrees well with the calculation from CFD model (3.13E+04 Pa). 


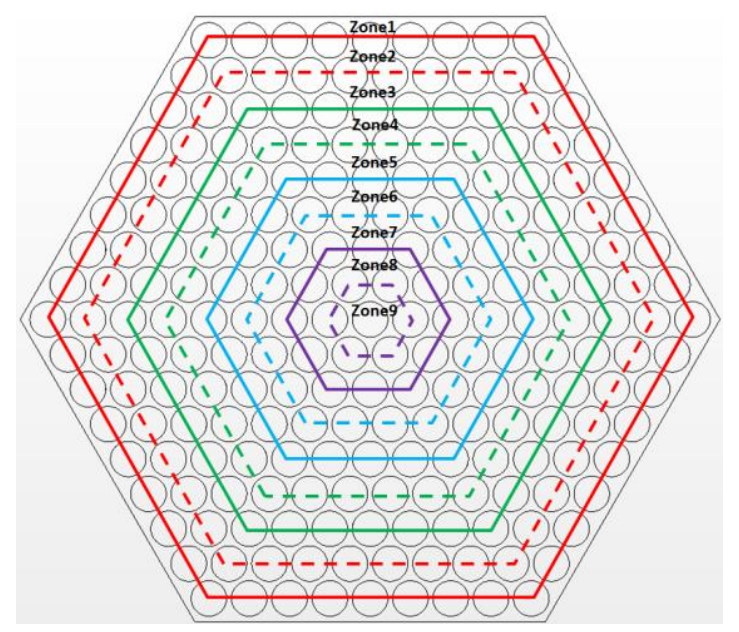

Figure 5 Sketch of 9 zones

In order to study the volumetric power distribution, the whole assembly is divided into 9 zones. The shape and the location of these 9 zones are shown in Figure 5. Zone 1 is located at the peripheral layer of the assembly and Zone 9 is located at the center of the assembly. The rest regions are located in between these two regions with same space interval. The volumetric heat flux $\mathrm{Q}_{\mathrm{v}}\left(\mathrm{W} / \mathrm{m}^{3}\right)$, in each region is calculated with the following formula:

$$
\mathrm{Q}_{\mathrm{v}}=\frac{\mathrm{N}_{\mathrm{r}} \cdot \mathrm{S} \cdot \mathrm{Q}_{\mathrm{S}}}{\mathrm{V}}
$$

where $N_{r}$ is the number of rods in each region, $S$ is the surface area of each rod $\left(\mathrm{m}^{2}\right), Q_{S}$ is the heat flux on the surface of each $\operatorname{rod}\left(\mathrm{W} / \mathrm{m}^{2}\right)$, and $\mathrm{V}$ is the volume of each zone. The flat-to-flat distance of the assembly is L. The detailed volumetric heat flux is listed in Table 2. It is quite remarkable that the volumetric heat flux in zone 1 is different from that in other zones. Based on the correlations and calculation mentioned above, three porous medium models are proposed.

Table 2 Volumetric heat flux in 9 zones

\begin{tabular}{|c|c|c|c|}
\hline Parameter & number of rods & Volume (V) & $25 Q_{S} S /\left(\sqrt{3} L^{2} / 2-96 \sqrt{3} P^{2}\right) H$ \\
\hline Zone 1 & 25 & $\left(\sqrt{3} L^{2} / 2-96 \sqrt{3} P^{2}\right) H$ & $2 \sqrt{3} Q_{S} S / 3 P^{2} H$ \\
\hline Zone 2 & 45 & $45 \sqrt{3} P^{2} H / 2$ & $2 \sqrt{3} Q_{S} S / 3 P^{2} H$ \\
\hline Zone 3 & 39 & $39 \sqrt{3} P^{2} H / 2$ & $2 \sqrt{3} Q_{S} S / 3 P^{2} H$ \\
\hline Zone 4 & 33 & $33 \sqrt{3} P^{2} H / 2$ & $2 \sqrt{3} Q_{S} S / 3 P^{2} H$ \\
\hline Zone 5 & 27 & $27 \sqrt{3} P^{2} H / 2$ & $2 \sqrt{3} Q_{S} S / 3 P^{2} H$ \\
\hline Zone 6 & 21 & $21 \sqrt{3} P^{2} H / 2$ & $2 \sqrt{3} Q_{S} S / 3 P^{2} H$ \\
\hline Zone 7 & 15 & $15 \sqrt{3} P^{2} H / 2$ & $2 \sqrt{3} Q_{S} S / 3 P^{2} H$ \\
\hline Zone 8 & 9 & $9 \sqrt{3} P^{2} H / 2$ & $3 \sqrt{3} P^{2} H / 2$ \\
\hline Zone 9 & 3 & $2 \sqrt{3} Q_{S} S / 3 P^{2} H$ & \\
\hline
\end{tabular}

Table 3 Parameters in three porous medium models

\begin{tabular}{|c|c|c|c|}
\hline Parameter & Porous Medium Model 1 & Porous Medium Model 2 & Porous Medium Model 3 \\
\hline $\begin{array}{c}\text { Velocity } \\
(\mathrm{m} / \mathrm{s})\end{array}$ & $V_{- \text {Regionl }}=1.59 \mathrm{~m} / \mathrm{s}$ & $\begin{array}{l}V_{- \text {Region } 1}=1.59 \mathrm{~m} / \mathrm{s} \\
V_{- \text {Region } 2}=1.59 \mathrm{~m} / \mathrm{s}\end{array}$ & $\begin{array}{l}V_{- \text {Region } 1}=1.44 \mathrm{~m} / \mathrm{s} \\
V_{- \text {Region } 2}=2.39 \mathrm{~m} / \mathrm{s}\end{array}$ \\
\hline $\begin{array}{c}\text { Porosity } \\
(-)\end{array}$ & $\chi_{\text {Region } 1}=0.319$ & $\begin{array}{l}\chi_{\_} \text {Region } 1=0.319 \\
\chi_{\text {Region } 2}=0.319\end{array}$ & $\begin{array}{l}\chi_{\text {Region } 1}=0.29 \\
\chi_{\text {Region } 2}=0.48\end{array}$ \\
\hline $\begin{array}{c}\text { Volumetric Heat Flux } \\
\left(\mathrm{WM} / \mathrm{m}^{3}\right)\end{array}$ & $Q_{v_{-} \text {Regionl } 1}=388.05$ & $\begin{array}{l}Q_{v_{-} \text {Region } 1}=404.63 \\
Q_{v_{-} \text {Region } 2}=295.14\end{array}$ & $\begin{array}{l}Q_{v_{-} \text {Region } 1}=404.63 \\
Q_{v_{-} \text {Region } 2}=295.14\end{array}$ \\
\hline Inertial Resistance $\left(\mathrm{kg} / \mathrm{m}^{4}\right)$ & $f_{- \text {Region } 1}=3172$ & $\begin{array}{l}f_{- \text {Region } 1}=3791 \\
f_{- \text {Region } 2}=1596\end{array}$ & $f_{- \text {Region } 1}=3791 f_{- \text {Region } 2}=1596$ \\
\hline
\end{tabular}




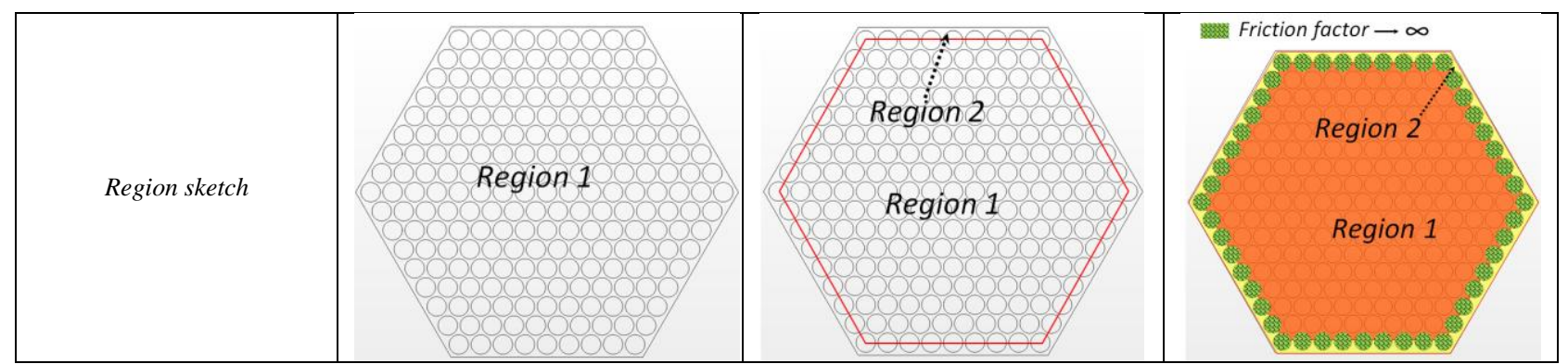

In the porous medium model 1 , the whole assembly is taken as one porous medium region with uniform volumetric heat flux. The porosity and velocity for the whole region is also set as uniform based on the ratio of open volume to total volume of the porous medium. The resistance for the whole region is calculated with the equation $1 \sim 4$.

In the porous medium model 2, the whole assembly is taken as a combination of two regions. Zone 1 in figure 5 is taken as a separate region due to its different volumetric heat flux. The remaining interior zones are taken as another region. Two regions are imposed with respective volumetric heat flux listed in Table 3 and respective resistance calculated with the equation 1 4. The porosity and velocity for these two regions use averaged value for the whole assembly like porous medium model 1 does.

In the porous medium model 3, region partition and volumetric heat flux for each region is the same as that in the porous medium model 2. However, the porosity and inlet velocity in these two regions are also distinct. Above all, the resistance is specified elaborately in the peripheral region (region 2). The resistance at the location of the rod and the wire in the peripheral region is specified as infinite while the resistance for the remainder of the domain it is calculated with the equation 1 4. A general concept of this model is to make the property of the porous medium as close to the actuality as possible without further increasing the computational cost.

In general, the porous media model requires less computational cost than that required for CFD model. Even so, the computational cost can be further reduced if we optimized the mesh strategy. Since the duct wall temperature is of interest, a higher mesh resolution close to the wall is preferred. However, the mesh resolution of the center region is refined along with that of the peripheral region with typical mesh strategy (Figure 6a), which results in the extra unnecessary computational cost. An Optimized mesh strategy is developed for the porous medium model to refine the mesh of the peripheral region without increasing the mesh density of the center region, as shown in Figure $6 \mathrm{c}$ and Figure 6d. In order to balance between the computational cost and the accuracy of the porous medium model, an intensive mesh sensitivity study is very necessary. The mesh sensitivity study is performed by adjusting the cell number in three direction i.e., circumferential direction, radial direction and axial direction. The cell number in these three directions for one pin is identified as a, b, c respectively (Figure 6b). The total cells count and detail parameters for the mesh sensitivity study are listed in Table 4.

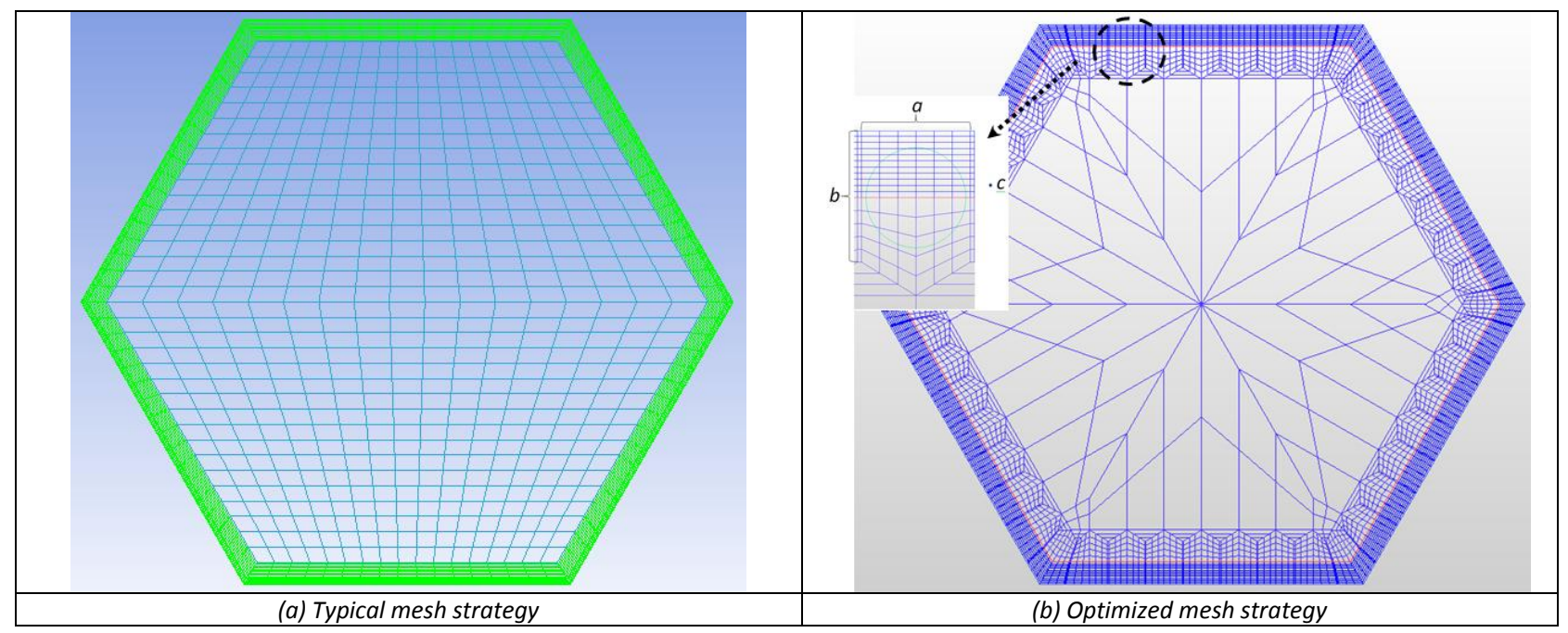




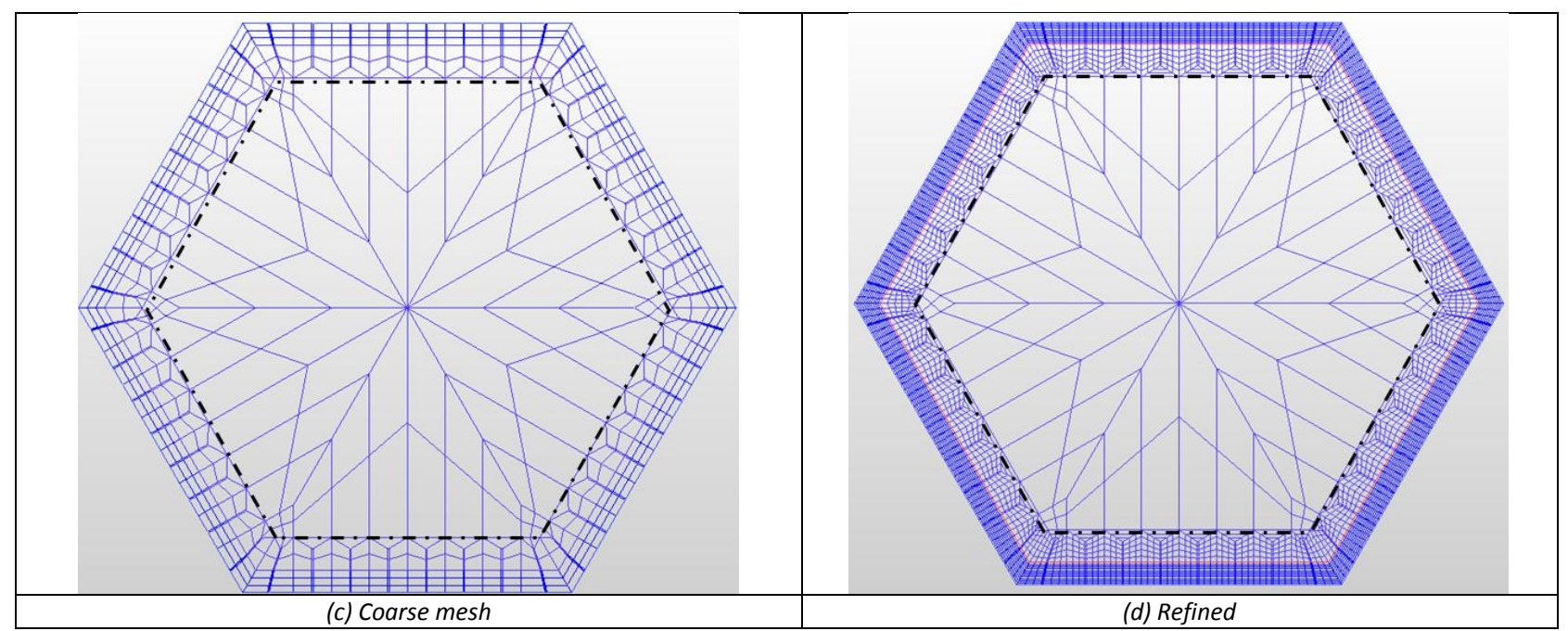

Figure 6 Mesh structure of the porous medium model

Table 4 Mesh parameters for sensitivity study

\begin{tabular}{|c|c|c|c|c|c|c|c|c|c|c|c|c|c|c|}
\hline \multicolumn{5}{|c|}{ Mesh in axial direction } & \multicolumn{5}{|c|}{ Mesh in circumferential direction } & \multicolumn{5}{|c|}{ Mesh in radial direction } \\
\hline \# & $a$ & $b$ & $c$ & Cell number & \# & $a$ & $b$ & $c$ & Cell number & $\#$ & $a$ & $b$ & $c$ & Cell number \\
\hline 1 & 12 & 12 & 10 & 52164 & 1 & 4 & 12 & 20 & 32604 & 1 & 8 & 9 & 20 & 51528 \\
\hline 2 & 12 & 12 & 20 & 110124 & 2 & 8 & 12 & 20 & 71364 & 2 & 8 & 12 & 20 & 71364 \\
\hline 3 & 12 & 12 & 50 & 284004 & 3 & 12 & 12 & 20 & 110124 & 3 & 8 & 18 & 20 & 111036 \\
\hline 4 & 12 & 12 & 100 & 573804 & 4 & 24 & 12 & 20 & 226404 & 4 & 8 & 24 & 20 & 150708 \\
\hline
\end{tabular}

The porous medium model 3 is employed for the mesh sensitivity study due to its highest requirement on the mesh density among three porous medium models. Figure 7 shows the duct wall temperature predictions on a line close to the outlet with different mesh resolutions, where $L_{h}$ is the length of the line. Mesh sensitivity study in axial direction indicates that the duct wall temperature is no more sensitive to the cell number in axial direction as long as $\mathrm{c}$ is more than 50. Given that the mesh of $\mathrm{c}=20$ also provides reasonable duct wall temperature with about half less computational cost, 20 nodes is considered to be an appropriate resolution in axial direction. Based on the sensitivity study in other directions, a mesh resolution of $a=8, b=12 c=20$ for one pin and total cell number 71,364 for the whole assembly is chosen to be the best solution for the porous medium model.

For one assembly of 217 pins, we need about 20 core hours to reach good convergence with CFD model while it only takes the porous medium model 0.1 core hours which is 200 times less. Given that the coupled and transient calculations are needed for accurately simulating the radial core expansion, the CFD model is no more possible for the calculation for whole core which include 199 assemblies due to unaffordable computational cost. On that point, the porous medium model is a good substitute. 


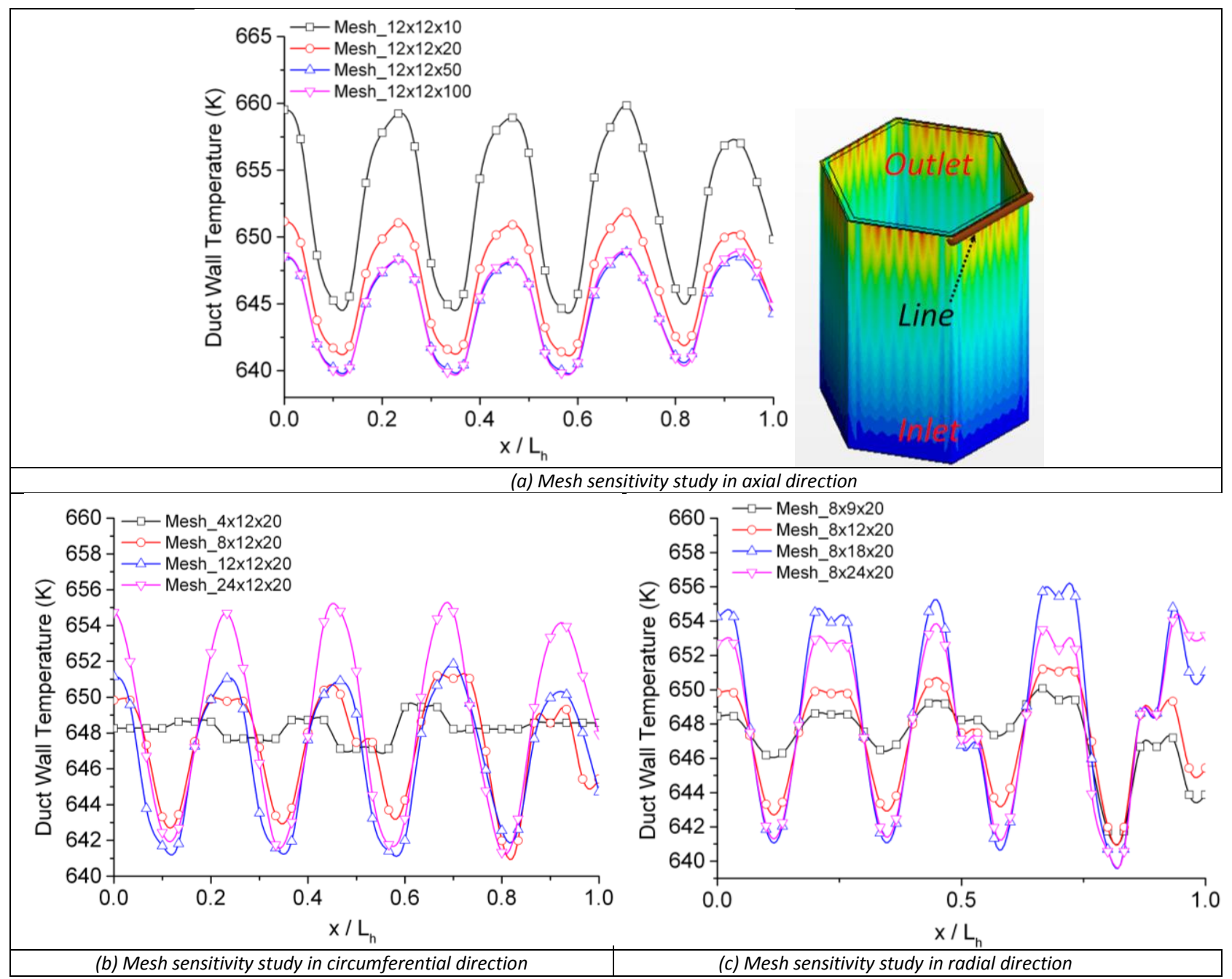

Figure 7 Mesh sensitivity study for porous medium model 3

\section{RESULTS AND DISCUSSION}

Three porous medium models are employed to predict the duct wall temperature for both bare rod bundle and wire wrapped rod bundle. The results are compared with the data from the CFD calculations for model validation. 


\subsection{Bare rod bundle}

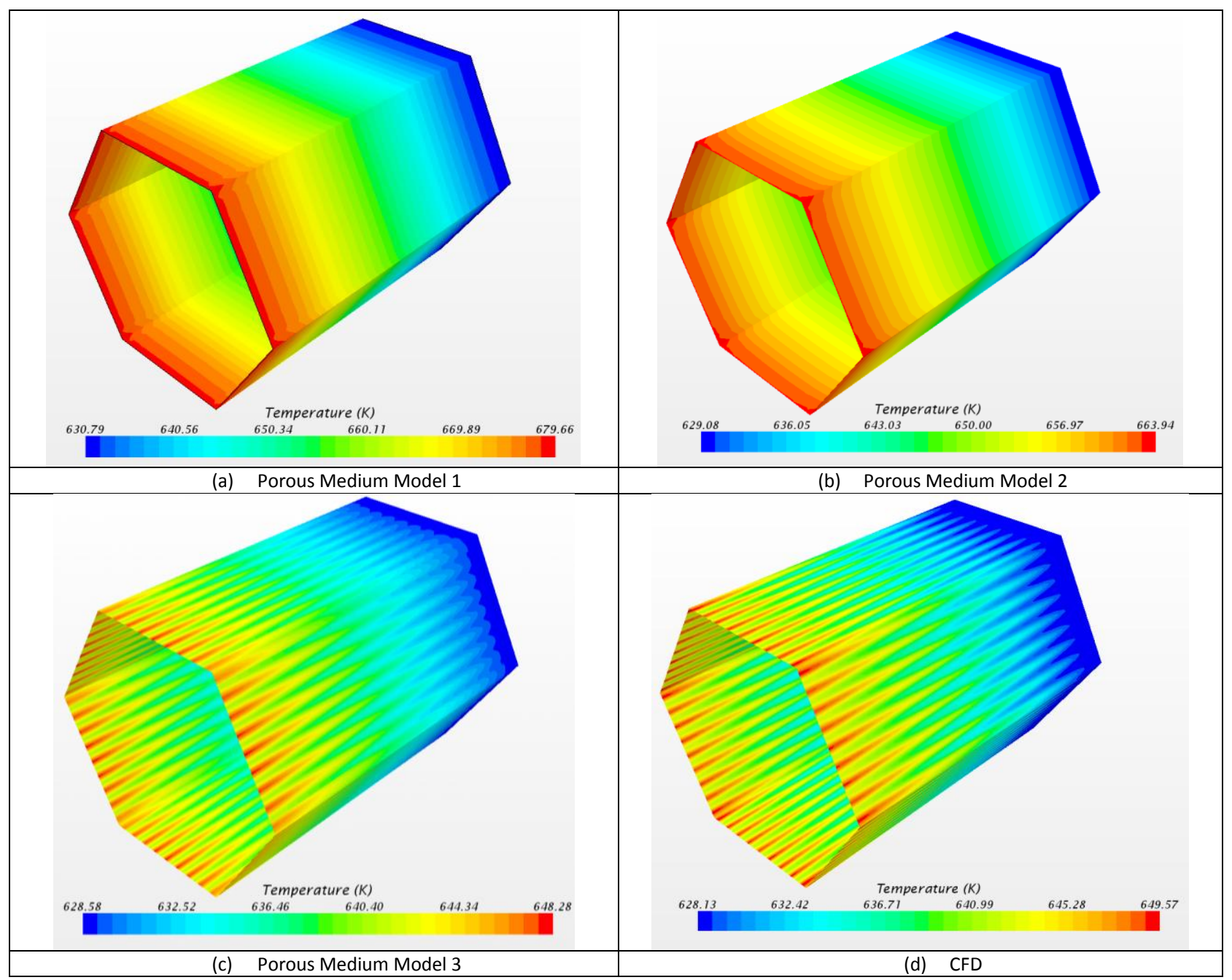

Figure 8 Contours of duct wall temperature with porous medium models and CFD model for bare rod bundle

Figure 8 shows the contours of the duct wall temperature from different models. Porous medium model 1 and 2 predict the similar duct wall temperature distribution, which show a flat temperature distribution along the flat wall surface and a higher duct wall temperature at the corner. It is because that the shear stress at the corner is higher than that along the flat wall, which results in lower velocity distribution at the corner. Since the fluid with lower velocity takes away less energy, the duct wall temperature at the corner is higher. However, in the CFD calculation, the duct wall temperature at the corner is lower. That is because there is no heat source in the very vicinity of the duct wall. The fluid with lower velocity at the corner transfers less energy from the heat source to the duct wall, which leads to a lower duct wall temperature. In another word, porous medium model 1 and 2 failed to predict the hot spot on the duct wall. In addition, Porous medium model 1 and 2 over predict the maximum duct wall temperature by $30^{\circ} \mathrm{C}$ and $14^{\circ} \mathrm{C}$ respectively. Porous medium model 2 has closer duct wall temperature prediction than Porous medium model 1 does because of a more precise volumetric heat flux distribution. The duct temperature predicted by porous medium model 3 ranges from $628 \mathrm{~K}$ to $648 \mathrm{~K}$, which agrees well with the range obtained by CFD calculation, i.e., from $628 \mathrm{~K}$ to $649 \mathrm{~K}$. It is noticeable that the isolines of the duct wall temperature in the CFD model present obvious serrations which are due to different flow resistance along the wall. These serrations are also well captured by porous medium model 3 . 


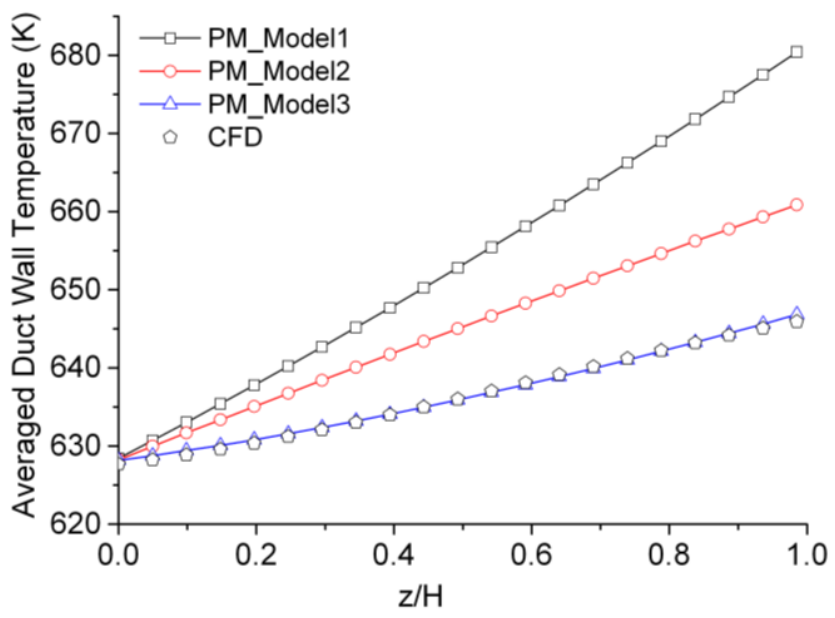

Figure 9 Averaged duct wall temperature distribution in axial direction for bare rod bundle

The averaged duct wall temperature along axial direction is shown in Figure 9. Porous medium model 1 and 2 over predict the averaged duct wall temperature all over the computational domain. Moreover, the discrepancy increases with the elevation, which indicates that the error is accumulated along the flow direction. However, the averaged duct wall temperature predicted by porous medium model 3 has achieved excellent agreement with the CFD calculation.

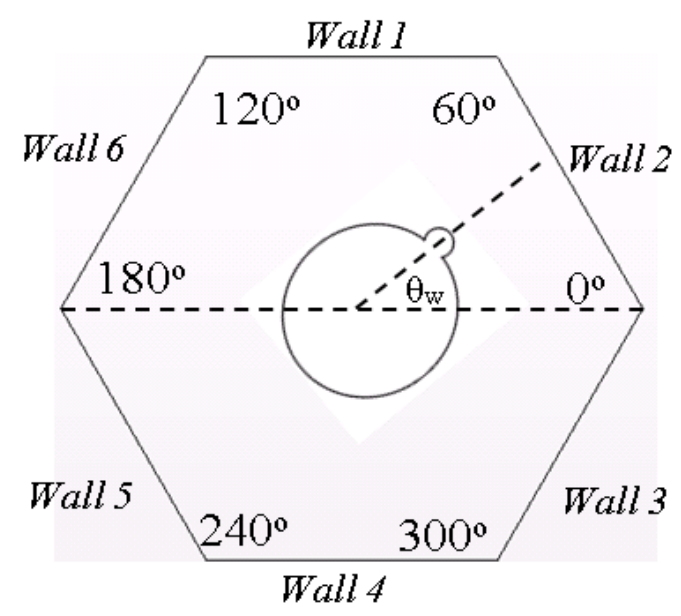

Figure 10 Definition of the angular positions

For purpose of conducting a more detailed analysis on the duct wall temperature, both a numbering and an angular scheme for the duct wall are made for better location identification (Figure 10). Figure 11 shows the circumferential duct temperature on different elevations. Porous medium model 3 captures the location for both peak and trough duct wall temperature in circumferential direction. At the corner of the duct (i.e., angle $=60,120,180$, $240,300)$, the temperature is lower due to greater shear stress there, which is also well reproduced by the porous medium model. The under estimation on the trough value of duct wall temperature by porous medium model is probably because of not being able to reproduce the velocity distribution accurately in the vicinity of the wall. 


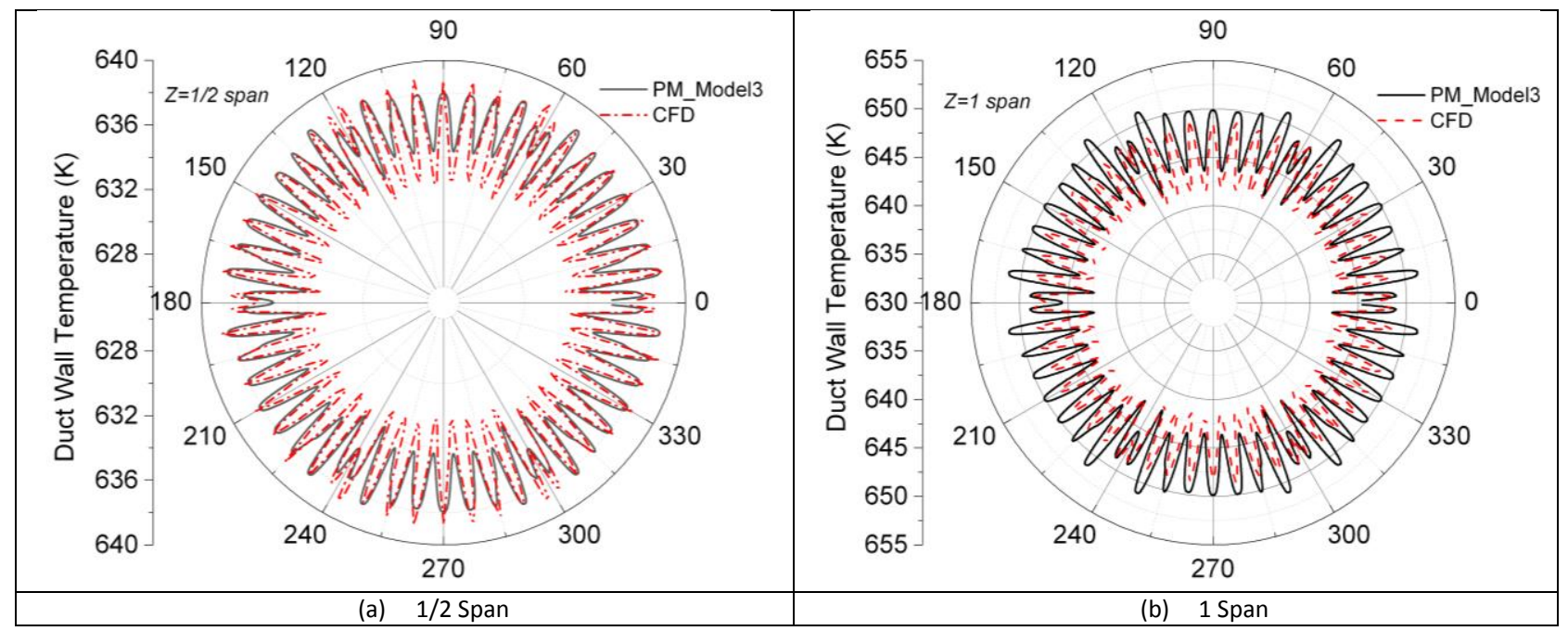

Figure 11 Comparisons of duct wall temperature between CFD model and Porous medium model in circumferential direction for bare rod bundle

Porous medium model 3 also achieves a reasonable agreement with the maximum and minimum duct wall temperature with the CFD calculation, as shown in Figure 12a, which indicates that porous medium model 3 can predict the location of the hot spot on the duct wall accurately. But some discrepancies are still found on the standard deviation of duct wall temperature prediction (Figure 12b). The standard deviation of duct wall temperature shows a nonlinear variation trend along the elevation with the CFD model, which is more linear with the porous medium model. Smaller standard deviation of duct wall temperature with the porous medium model means under estimation on temperature difference of the duct wall. Since the maximum discrepancy of the standard deviation which appears in the center of the domain is only about $1{ }^{\circ} \mathrm{C}$, the confidence in relying on the porous medium model to predict the duct wall temperature in bare rod bundle is not lost.

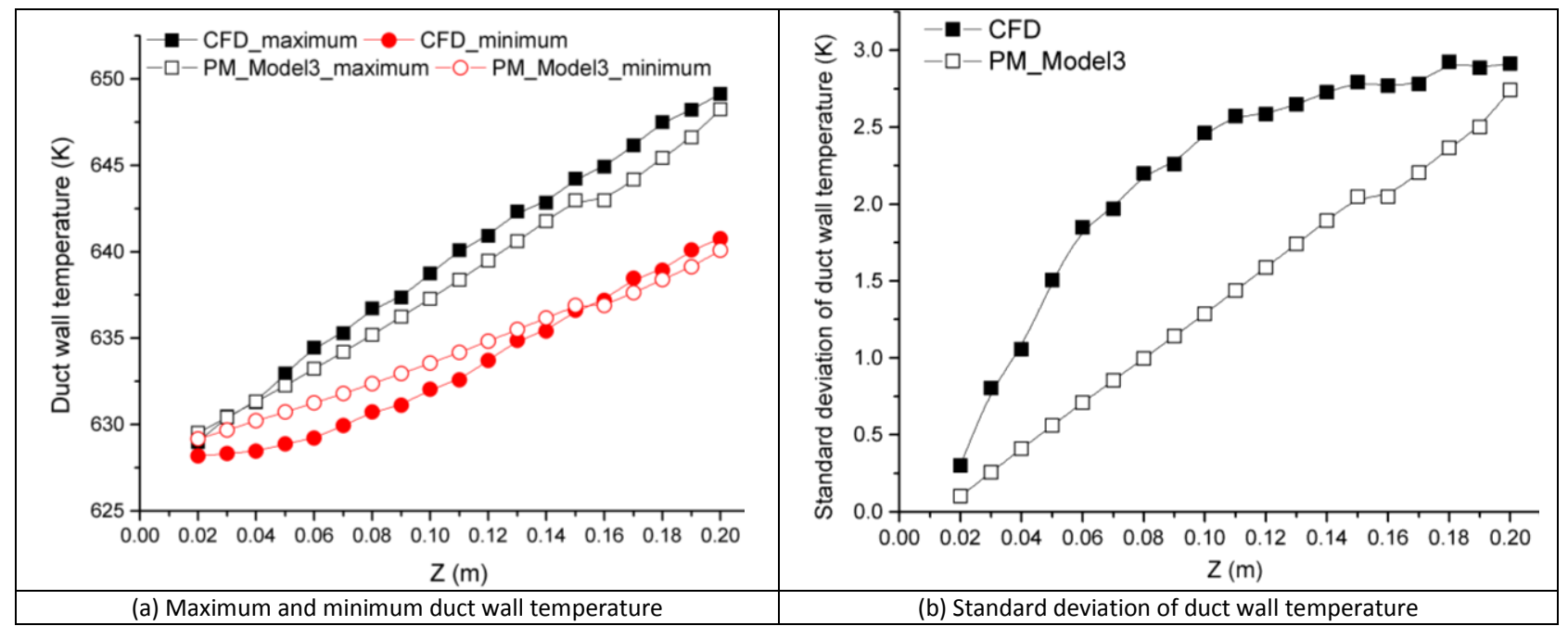

Figure 12 Maximum, minimum and standard deviation of the duct wall temperature in axial direction for bare rod bundle

\subsection{Wire wrapped rod bundle}




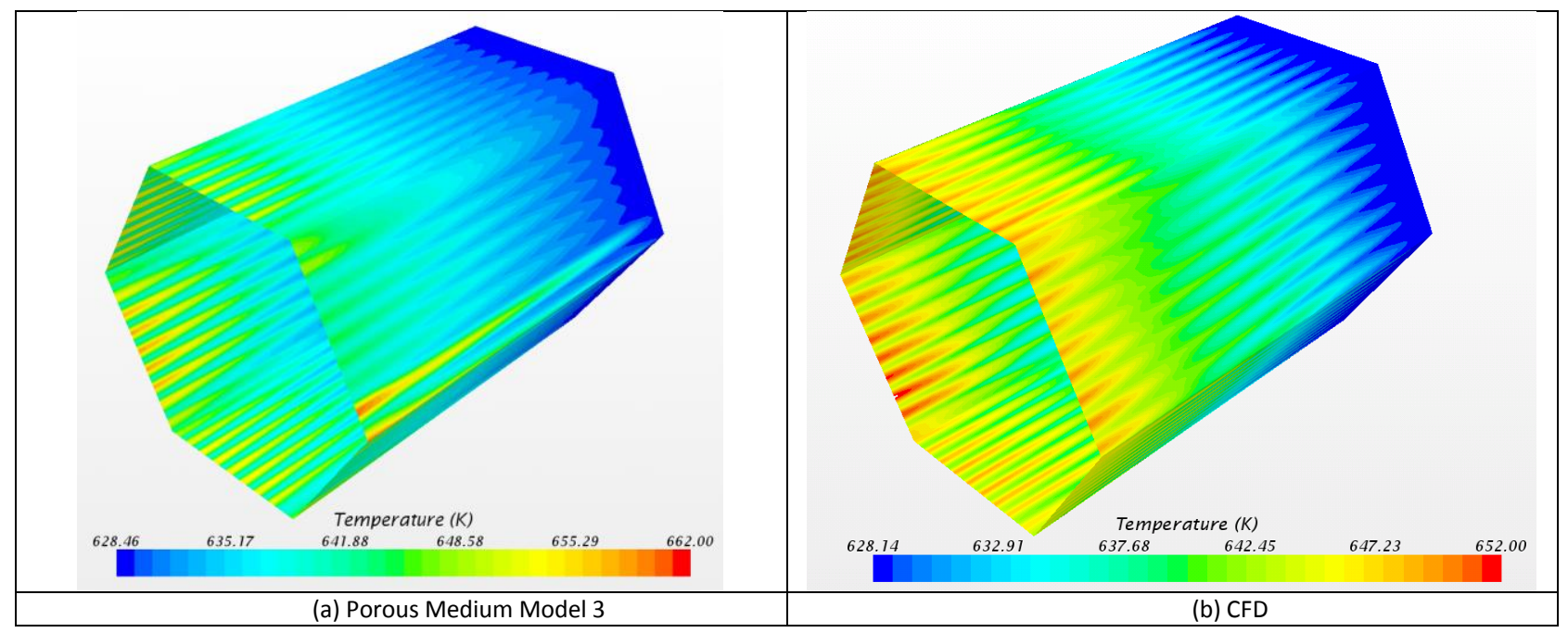

Figure 13 Contours of duct wall temperature with porous medium model 3 and CFD model for wire wrapped rod bundle

Since porous medium model 1 and porous medium model 2 has no capacity of taking into account the wire effect, only porous medium model 3 is employed for wire wrapped rod bundle. Figure 13 shows the contours of the duct wall temperature for wire wrapped rod bundle. The non-uniformity of the duct wall temperature is found in both CFD and porous medium model. The porous medium model overestimates the maximum duct wall temperature by $10{ }^{\circ} \mathrm{C}$.

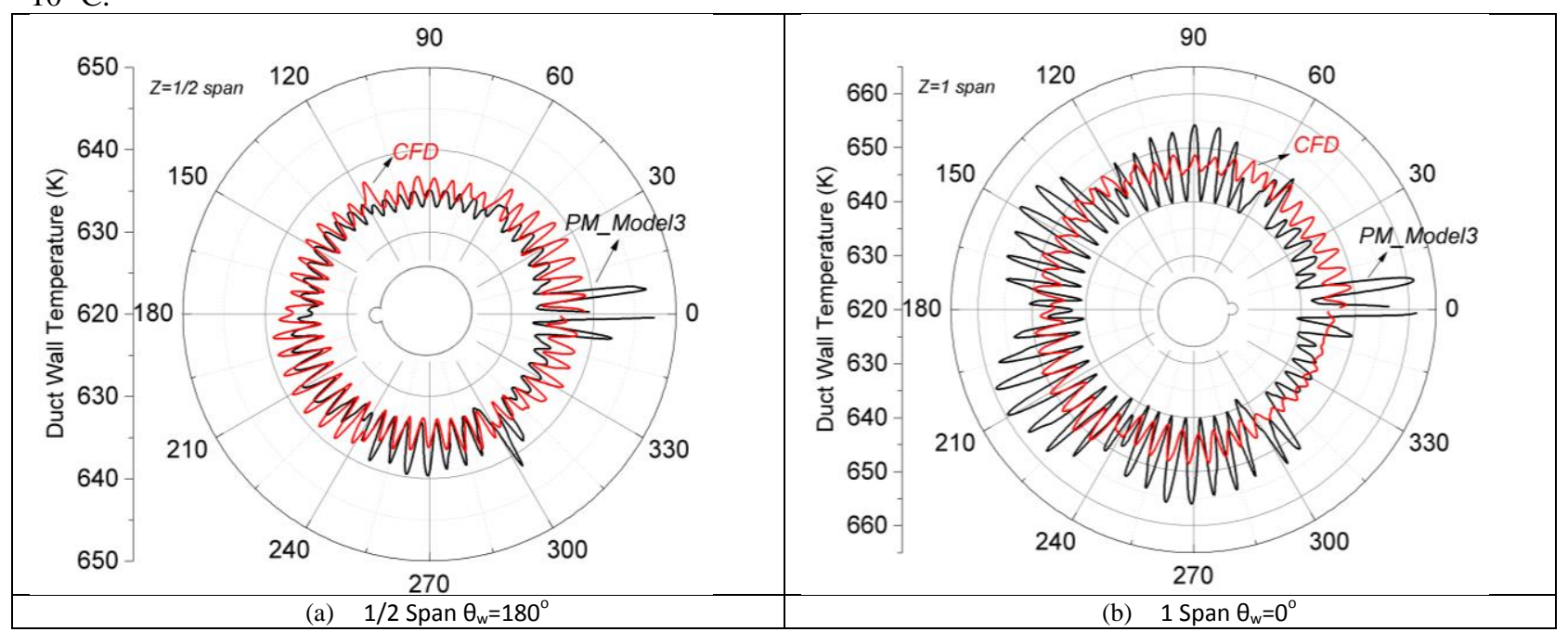

Figure 14 Comparisons of duct wall temperature between CFD model and porous medium model in circumferential direction for wire wrapped rod bundle

The wire angle $\theta \mathrm{w}$ is defined in figure 10 to identify the relative location between wire and duct wall. At $1 / 2 \mathrm{~s}$ pan, where $\theta \mathrm{w}$ is $180^{\circ}$, the location of the peak values from CFD model and porous medium model shows some mismatch from $0^{\circ} \mathrm{C}$ to $120^{\circ} \mathrm{C}$ (wall1 and wall2). At 1 span, where $\theta \mathrm{w}$ is $0^{\circ}$, the mismatch is less obvious. In addition, a temperature reduction from $300^{\circ} \mathrm{C}$ to $360^{\circ} \mathrm{C}$ is well captured by the porous medium model. (Figure 14) 


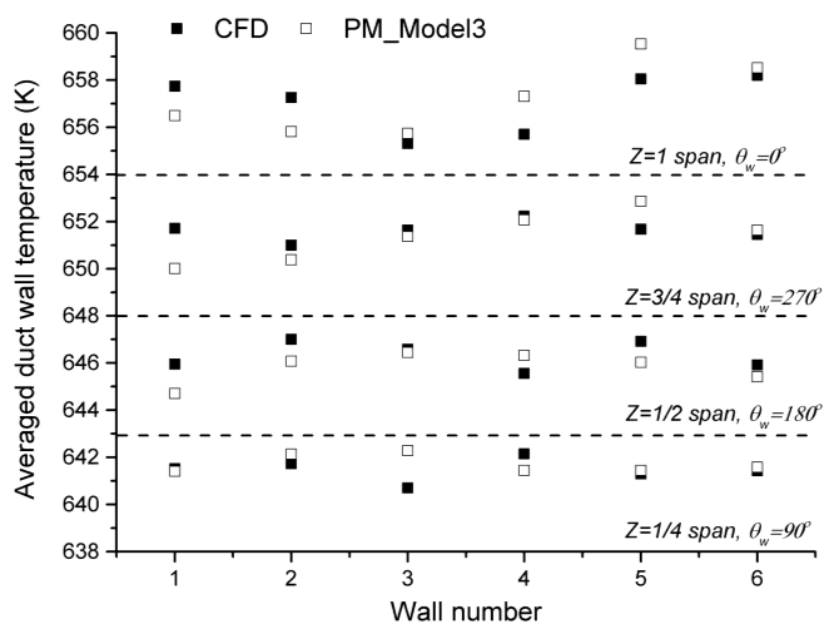

Figure 15 Averaged wall temperatures for each duct wall on different elevations

In order to investigate the wire effect on the duct wall temperature, the average temperatures for each wall on different elevations are shown in Figure 15. No obvious rule on temperature enhancement or reduction of the wall is found when the wires face to that wall which shrink the flow pass. It suggests that the wire effect is not only local but also global. The wires not only shrink the flow pass but also change the flow distribution near the wall. Since the porous medium model only takes into account the wire effect in the peripheral region, it is inevitable to miss some global wire effect. For instance, the porous medium model predicts the wall temperature difference due to the wire effect near the outlet ( $\mathrm{z}=1$ span) well but fails to predict it near the inlet $(\mathrm{z}=1 / 4$ span), especially for duct wall 3 . Therefore, it is worthwhile developing a model to describe the global wire effect in the porous medium model. However, the wire adds more complexity on the flow characteristics of rod bundle assembly in flow direction, which means that a refined mesh for the porous medium model is needed to capture these features.

\section{CONCLUSIONS}

A novel class of porous medium models has been established for predicting SFR duct wall temperature profiles. This class of models is 400 times less computationally expensive than conventional CFD simulations that explicitly represent the wire-wrap and fuel pin geometry. It is also significantly less computationally expensive than CFD simulations based on momentum source models. Moreover, the new class of models showed very good agreement with detailed CFD simulations, while conventional porous medium models exhibited poor agreement. In particular, porous medium 3 which divides the assembly into distinct regions and employs a precise resistance distribution in the outmost region reproduces the duct wall temperature of the CFD simulation with an excellent precision for bare rod bundle and reasonably well for wire-wrapped rod bundles. In fact, although the overall results remain within $2^{\circ} \mathrm{C}$ of the detailed CFD predictions, some global wire effect are not captured well by the current model. In further research, a model could be developed to take better into account the global wire effect. Following the results of this study, porous medium model 3 will become the standard for future work in SHARP with porous media. It is also a very promising tool for coupled transient calculation for radial core expansion.

\section{ACKNOWLEDGMENTS}

The submitted manuscript has been created by UChicago Argonne, LLC, Operator of Argonne National Laboratory ("Argonne"). Argonne, a U.S. Department of Energy Office of Science laboratory, is operated under Contract No. DE-AC02-06CH11357. The U.S. Government retains for itself, and others acting on its behalf, a paid- 
up nonexclusive, irrevocable worldwide license in said article to reproduce, prepare derivative works, distribute copies to the public, and perform publicly and display publicly, by or on behalf of the Government.

\section{REFERENCES}

Fontana, M., 1973. Temperature distribution in the duct wall and at the exit of a19-rod simulated LMFBR Fuel Assembly (FFM-2A). In: ORNL-4852, Oak Ridge National Laboratory, TN.

Wantland, J., Gnadt, P., MacPherson, R., Fontana, M., Hanus, N., Smith, C., 1976. The effects of duct configuration on flow and temperature structure in sodium-cooled 19-rod simulated LMFBR fuel bundles with helical wire-wrap spacers. In: Sixteenth National Heat Transfer Conference, St. Louis, MO.

Engel, F., Minushkin, B., Atkins, R., Markley, R., 1980. Characterization of heat transfer and temperature distribution in an electrically heated model of an LMFBR blanket assembly. Nucl. Eng. Des. 62, 335-347

Choi, S., Choi, K., Nam, H., Choi, J., Choi, H., 2003. Measurement of pressure drop in a full-scale fuel assembly of a Liquid Metal Reactor. J. Pressure Vessel Technol.125.

Sato, H., Kobayashi, J., Miyakoshi, H., Kamide, H., 2009. Study on velocity field ina wire wrapped fuel pin bundle of sodium cooled reactor - detailed velocitydistribution in a subchannel. In: NURETH13, Kanazawa, Japan

Prakash, V., Thirumalai, M., Anandaraj, M., Anup Kumar, P., Ramdasu, D., Pandey,G., Padmakumar, G., Anandbabu, C., Kalyanasundaram, P., 2011. Experimental qualification of subassembly design for prototype Fast Breeder Reactor. Nucl.Eng. Des. 241, 3325-3332.

Collingham, R., Hill, V., Yatabe, J., Thorne, W., 1970. Developments and results of an electrically heated seven-pin bundle assembly for thermal hydraulic testing in liquid metals. In: BNWL-SA-3286, Batelle North-West Labatories, WA.

Won-Seok Kim, Young-Gyun Kim, Young-Jin Kim, A subchannel analysis code MATRA-LMR for wire wrapped LMR subassembly, Annals of Nuclear Energy, Volume 29, Issue 3, February 2002, Pages 303-321

Fanning, T. H., W. D. Pointer, and J. W. Thomas. "Multi-resolution modeling of subassembly pin bundles for advanced fast reactor safety simulations." Proceedings of M\&C 2009 (2009).

Wheeler, C. L., et al. COBRA-IV-I: An interim version of COBRA for thermal-hydraulic analysis of rod bundle nuclear fuel elements and cores. No. BNWL-1962. Battelle Pacific Northwest Labs., Richland, Wash.(USA), 1976.

Stewart, Charles W., et al. COBRA-IV: The model and the method. No. BNWL-2214. Pacific Northwest Lab., Richland, WA (USA), 1977.

Basehore, K. L., and N. E. Todreas. SUPERENERGY-2: a multiassembly, steady-state computer code for LMFBR core thermal-hydraulic analysis. Battelle Pacific Northwest Labs., Richland, WA (USA), 1980.

Yang, W. S., and A. M. Yacout. Assessment of the SE2-ANL code using EBR-II temperature measurements. Argonne National Lab., IL (United States). Funding organisation: USDOE, Washington, DC (United States), 1995.

E. Merzari, H. Ninokata, Proper orthogonal decomposition of the flow in a tight lattice rod-bundle, Nuclear Engineering and Design, Volume 241, Issue 11, 2011, Pages 4621-4632

X. Cheng, Y.Q. Yu, Local thermal-hydraulic behaviour in tight 7-rod bundles, Nuclear Engineering and Design, Volume 239, Issue 10,2009, Pages 1944-1955

Fischer, P., Lottes, J., Siegel, A., Palmiotti, G., 2007. Large eddy simulation of wire-wrapped fuel pins I: hydrodynamics of a single pin. In: M and C+SNA 2007,Monterey, CA.

Merzari, E., Pointer, W., Smith, J., Fischer, P., 2010. Numerical simulation of the flow in wire-wrapped pin bundles: effect of pin-wire contact modeling. In: CFD4NRS-3Workshop, Washington, DC.

Pointer, W., Fischer, P., Smith, J., Obabko, A., Siegel, A., 2009. Simulation of turbu-lent diffusion in wire-wrapped Sodium Fast Reactor Fuel Assemblies. In: FR-09,Kyoto, Japan.

Hu, R., Fanning, T., 2010. Intermediate-resolution method for thermal-hydraulicsmodeling of a wire-wrapped pin bundle. Trans. Am. Nucl. Soc. 103, 1003-1005.

Y.I. Chang, et.al., Advanced Burner Test Reactor preconceptual design report, ANL-ABR-1 (ANL-AFCI-173), Argonne National Laboratory (2006)

V.S. Mahadevan, E. Merzari, T. Tautges, R. Jain, A. Obabko, M. Smith, P. Fischer, High-resolution coupled physics solvers for analyzing fine-scale nuclear reactor design problems, Philosophical transactions of the Royal Society A. 372, 2021 20130381(2014) 
E.U. Khan, W.M. Rohsenow, A.A. Sonin, N.E. Todereas, A Porous Body Model for Predicting Temperature Distribution in Wire-Wrapped Fuel Rod Assemblies, Nuclear Engineering and Design. 35, 1-12 (1975)

K. Rehme, Pressure Drop Correlations for Fuel Element Spacers. Nuclear Technology 17, 15-23. (1973)

R.A.Wigeland, Effect of a Detailed Radial Core Expansion Reactivity Feedback Model on ATWS Calculations Using SASSYS/SAS4A,1986,Trans.AM.Nucl.Soc.,53, 303

Y. Yu, E. Merzari, and J. Thomas, "SFR Duct Wall Temperature Prediction with a Porous Medium Model," Transactions of the American Nuclear Society, Vol. 111, 1651-1653, (2014).

Y.Q. Yu, B.H. Yan, X. Cheng, H.Y. Gu, Simulation of turbulent flow inside different subchannels in tight lattice bundle, Ann. Nucl. Energy, 38 (2011), pp. 2363-2373 


\section{REFERENCES}

Fontana, M., 1973. Temperature distribution in the duct wall and at the exit of a19-rod simulated LMFBR Fuel Assembly (FFM-2A). In: ORNL-4852, Oak Ridge National Laboratory, TN.

Wantland, J., Gnadt, P., MacPherson, R., Fontana, M., Hanus, N., Smith, C., 1976. The effects of duct configuration on flow and temperature structure in sodium-cooled 19-rod simulated LMFBR fuel bundles with helical wire-wrap spacers. In: Sixteenth National Heat Transfer Conference, St. Louis, MO.

Engel, F., Minushkin, B., Atkins, R., Markley, R., 1980. Characterization of heat transfer and temperature distribution in an electrically heated model of an LMFBR blanket assembly. Nucl. Eng. Des. 62, 335-347

Choi, S., Choi, K., Nam, H., Choi, J., Choi, H., 2003. Measurement of pressure drop in a full-scale fuel assembly of a Liquid Metal Reactor. J. Pressure Vessel Technol.125.

Sato, H., Kobayashi, J., Miyakoshi, H., Kamide, H., 2009. Study on velocity field ina wire wrapped fuel pin bundle of sodium cooled reactor-detailed velocitydistribution in a subchannel. In: NURETH13, Kanazawa, Japan

Prakash, V., Thirumalai, M., Anandaraj, M., Anup Kumar, P., Ramdasu, D., Pandey,G., Padmakumar, G., Anandbabu, C., Kalyanasundaram, P., 2011. Experimental qualification of subassembly design for prototype Fast Breeder Reactor. Nucl.Eng. Des. 241, 3325-3332.

Collingham, R., Hill, V., Yatabe, J., Thorne, W., 1970. Developments and results of an electrically heated seven-pin bundle assembly for thermal hydraulic testing in liquid metals. In: BNWL-SA-3286, Batelle North-West Labatories, WA.

Won-Seok Kim, Young-Gyun Kim, Young-Jin Kim, A subchannel analysis code MATRA-LMR for wire wrapped LMR subassembly, Annals of Nuclear Energy, Volume 29, Issue 3, February 2002, Pages 303-321

Fanning, T. H., W. D. Pointer, and J. W. Thomas. "Multi-resolution modeling of subassembly pin bundles for advanced fast reactor safety simulations." Proceedings of M\&C 2009 (2009).

Wheeler, C. L., et al. COBRA-IV-I: An interim version of COBRA for thermal-hydraulic analysis of rod bundle nuclear fuel elements and cores. No. BNWL-1962. Battelle Pacific Northwest Labs., Richland, Wash.(USA), 1976.

Stewart, Charles W., et al. COBRA-IV: The model and the method. No. BNWL-2214. Pacific Northwest Lab., Richland, WA (USA), 1977.

Basehore, K. L., and N. E. Todreas. SUPERENERGY-2: a multiassembly, steady-state computer code for LMFBR core thermal-hydraulic analysis. Battelle Pacific Northwest Labs., Richland, WA (USA), 1980.

Yang, W. S., and A. M. Yacout. Assessment of the SE2-ANL code using EBR-II temperature measurements. Argonne National Lab., IL (United States). Funding organisation: USDOE, Washington, DC (United States), 1995.

E. Merzari, H. Ninokata, Proper orthogonal decomposition of the flow in a tight lattice rod-bundle, Nuclear Engineering and Design, Volume 241, Issue 11, 2011, Pages 4621-4632

X. Cheng, Y.Q. Yu, Local thermal-hydraulic behaviour in tight 7-rod bundles, Nuclear Engineering and Design, Volume 239, Issue 10,2009, Pages 1944-1955

Fischer, P., Lottes, J., Siegel, A., Palmiotti, G., 2007. Large eddy simulation of wire-wrapped fuel pins I: hydrodynamics of a single pin. In: M and C+SNA 2007, Monterey, CA.

Merzari, E., Pointer, W., Smith, J., Fischer, P., 2010. Numerical simulation of the flow in wire-wrapped pin bundles: effect of pin-wire contact modeling. In: CFD4NRS-3Workshop, Washington, DC.

Pointer, W., Fischer, P., Smith, J., Obabko, A., Siegel, A., 2009. Simulation of turbu-lent diffusion in wire-wrapped Sodium Fast Reactor Fuel Assemblies. In: FR-09,Kyoto, Japan.

Hu, R., Fanning, T., 2010. Intermediate-resolution method for thermal-hydraulicsmodeling of a wire-wrapped pin bundle. Trans. Am. Nucl. Soc. 103, 1003-1005.

Y.I. Chang, et.al., Advanced Burner Test Reactor preconceptual design report, ANL-ABR-1 (ANL-AFCI-173), Argonne National Laboratory (2006)

V.S. Mahadevan, E. Merzari, T. Tautges, R. Jain, A. Obabko, M. Smith, P. Fischer, High-resolution coupled physics solvers for analyzing fine-scale nuclear reactor design problems, Philosophical transactions of the Royal Society A. 372, 2021 20130381(2014)

E.U. Khan, W.M. Rohsenow, A.A. Sonin, N.E. Todereas, A Porous Body Model for Predicting Temperature Distribution in Wire-Wrapped Fuel Rod Assemblies, Nuclear Engineering and Design. 35, 1-12 (1975)

K. Rehme, Pressure Drop Correlations for Fuel Element Spacers. Nuclear Technology 17, 15-23. (1973)

R.A.Wigeland, Effect of a Detailed Radial Core Expansion Reactivity Feedback Model on ATWS Calculations Using SASSYS/SAS4A,1986,Trans.AM.Nucl.Soc.,53, 303 